\title{
Brainstem Pain-Control Circuitry Connectivity in Chronic Neuropathic Pain
}

\author{
Emily P. Mills, ${ }^{1}$ Flavia Di Pietro, ${ }^{1}$ Zeynab Alshelh, ${ }^{1}$ Chris C. Peck, ${ }^{2}$ Greg M. Murray, ${ }^{2}$ E. Russell Vickers, ${ }^{1}$ \\ and ${ }^{\odot}$ Luke A. Henderson ${ }^{1}$ \\ ${ }^{1}$ Department of Anatomy and Histology and 2 Faculty of Dentistry, University of Sydney, Sydney 2006, New South Wales, Australia
}

\begin{abstract}
Preclinical investigations have suggested that altered functioning of brainstem pain-modulation circuits may be crucial for the maintenance of some chronic pain conditions. While some human psychophysical studies show that patients with chronic pain display altered pain-modulation efficacy, it remains unknown whether brainstem pain-modulation circuits are altered in individuals with chronic pain. The aim of the present investigation was to determine whether, in humans, chronic pain following nerve injury is associated with altered ongoing functioning of the brainstem descending modulation systems. Using resting-state functional magnetic resonance imaging, we found that male and female patients with chronic neuropathic orofacial pain show increased functional connectivity between the rostral ventromedial medulla (RVM) and other brainstem pain-modulatory regions, including the ventrolateral periaqueductal gray (vlPAG) and locus ceruleus (LC). We also identified an increase in RVM functional connectivity with the region that receives orofacial nociceptor afferents, the spinal trigeminal nucleus. In addition, the vlPAG and LC displayed increased functional connectivity strengths with higher brain regions, including the hippocampus, nucleus accumbens, and anterior cingulate cortex, in individuals with chronic pain. These data reveal that chronic pain is associated with altered ongoing functioning within the endogenous pain-modulation network. These changes may underlie enhanced descending facilitation of processing at the primary synapse, resulting in increased nociceptive transmission to higher brain centers. Further, our findings show that higher brain regions interact with the brainstem modulation system differently in chronic pain, possibly reflecting top-down engagement of the circuitry alongside altered reward processing in pain conditions.
\end{abstract}

Key words: analgesia; chronic pain; locus ceruleus; midbrain periaqueductal gray; rostral ventromedial medulla; spinal trigeminal nucleus

Significance Statement

Experimental animal models and human psychophysical studies suggest that altered functioning of brainstem pain-modulation systems contributes to the maintenance of chronic pain. However, the function of this circuitry has not yet been explored in humans with chronic pain. In this study, we report that individuals with orofacial neuropathic pain show altered functional connectivity between regions within the brainstem pain-modulation network. We suggest that these changes reflect largely central mechanisms that feed back onto the primary nociceptive synapse and enhance the transfer of noxious information to higher brain regions, thus contributing to the constant perception of pain. Identifying the mechanisms responsible for the maintenance of neuropathic pain is imperative for the development of more efficacious therapies.

\section{Introduction}

Experimental animal studies have revealed that the brainstem contains multiple neural circuits that can profoundly inhibit or

\footnotetext{
Received June 13, 2017; revised 0ct. 23, 2017; accepted Nov. 12, 2017.

Author contributions: C.C.P., G.M.M., E.R.V., and L.A.H. designed research; E.P.M., F.D.P., Z.A., E.R.V., and L.A.H. performed research; E.P.M. analyzed data; E.P.M., F.D.P., Z.A., C.C.P., G.M.M., E.R.V., and L.A.H. wrote the paper.

This work was supported by the National Health and Medical Research Council of Australia, Grants 1032072 and 1059182. We thank the many volunteers in this study.

The authors declare no competing financial interests.

Correspondence should be addressed to Luke A. Henderson, Department of Anatomy and Histology, F13, Univer-

sity of Sydney, Sydney 2006, NSW, Australia. E-mail: lukeh@anatomy.usyd.edu.au
}

facilitate incoming nociceptive information at the level of the primary afferent synapse. These descending modulatory circuits include direct and indirect descending projections to the dorsal horn and spinal trigeminal nucleus $(\mathrm{SpV})$ from such regions as the midbrain periaqueductal gray matter (PAG), the rostral ventromedial medulla (RVM), the locus ceruleus (LC), and the subnucleus reticularis dorsalis (SRD; Basbaum and Fields, 1984; Heinricher et al., 2009; Ossipov et al., 2010; Mason, 2012). In 
addition to a presumed role in modulating acute noxious stimuli, these circuits might be involved in the generation and maintenance of chronic pain, particularly that following nerve damage or presumed damage (i.e., chronic neuropathic pain), as suggested by recent preclinical data (Burgess et al., 2002; Pertovaara, 2013; Wang et al., 2013).

While it remains unknown whether the function of these brainstem modulatory circuits is altered in humans with chronic pain, multiple psychophysical investigations have suggested that they are. For example, some studies using conditioned pain modulation, in which the perceived pain intensity of one noxious stimulus is modified by another at a distant site, have provided evidence that the effectiveness of these pain-modulation circuits is significantly altered in individuals with various chronic pain conditions (Yarnitsky, 2010; Garrett et al., 2013; Nasri-Heir et al., 2015). There is evidence from experimental studies in both animals and humans that the effects of these pain-modulatory systems are mediated by structures in the brainstem, in particular the area of the SRD (Le Bars et al., 1979; De Broucker et al., 1990; Youssef et al., 2016). These studies strongly suggest that alterations in the ongoing functioning of brainstem nociceptive modulation systems may play a critical role in the initiation and/or the maintenance of various human chronic pain conditions.

To date, no study has focused on the ongoing function of brainstem nociceptive modulation circuits, including the PAG, RVM, LC, and SRD in humans with chronic pain. The aim of the present study was to use resting-state functional magnetic resonance imaging (fMRI) to explore the ongoing function of these brainstem circuits in individuals with painful trigeminal neuropathy (PTN), a chronic orofacial neuropathic pain disorder. We hypothesize that individuals with PTN would display enhanced fMRI signal covariation between regions involved in endogenous pain modulation, such as the ventrolateral PAG (vlPAG), RVM, LC, and SRD, and the region where orofacial nociceptive information enters the CNS, i.e., the SpV. Such changes may result in greater facilitation of ongoing activity within the $\mathrm{SpV}$, enhanced noxious information transfer to higher brain centers, and the constant perception of pain. Furthermore, these brainstem circuits can be engaged by higher brain regions, such as the orbitofrontal cortex and ventral striatum (Bandler et al., 2000; Floyd et al., 2000) and so we hypothesize that PTN subjects will display altered functional connectivity strengths between these rostral brain regions and the PAG and LC.

\section{Materials and Methods}

\section{Subjects and pain measures}

Twenty-four subjects with PTN [eight males; mean ( \pm SEM) age, $46 \pm 3$ years; range, $19-71$ years] and 46 pain-free subjects (17 males; age, $42 \pm$ 2 years; range, 22-66 years) were recruited for the study between 2011 and 2017. There was no significant difference in age ( $t$ test; $p>0.05)$ or gender distribution $\left(\chi^{2}\right.$ test, $\left.p>0.05\right)$ between the two subject groups. PTN subjects were recruited and diagnosed in accordance with the Liverpool criteria (Nurmikko and Eldridge, 2001) by clinicians (C.C.P, E.R.V, G.M.M) in the research group. Control participants were recruited by word of mouth. Informed written consent was obtained for all procedures, which were conducted with the approval of local institutional human research ethics committees and are consistent with the Declaration of Helsinki. Other data from several subjects used in this investigation have been used in previous studies (Gustin et al., 2011; Henderson et al., 2013; Wilcox et al., 2015a; Alshelh et al., 2016).

The PTN subjects assessed the intensity of their ongoing pain using a visual analog scale ( 0 , no pain, to 10 , worst pain imaginable) three times per day for 7 consecutive days during the week of the scanning session. The average of these pain ratings was taken as a measure of current pain

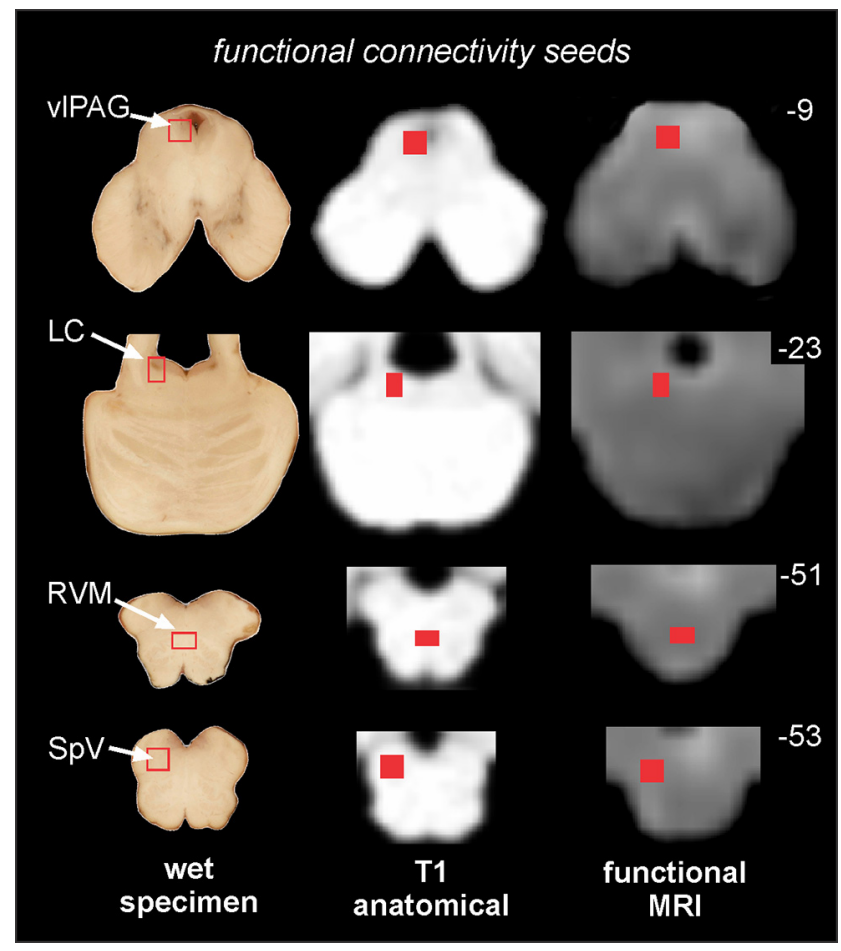

Figure 1. Locations of functional connectivity seeds overlaid onto axial slices of a wet specimen, a representative $T 1$-weighted anatomical image, and a representative brainstem fMRI image normalized to the SUIT template. Slice locations in MNI space are indicated at the top right. The present study focused on the following regions of interest: the midbrain vIPAG, the LC, the RVM, and the SpV.

intensity. Subjects also described their pain distribution by outlining the area of their chronic pain on a standard drawing of the face and described the quality of their ongoing pain by completing a McGill Pain Questionnaire (MPQ).

\section{MRI scans}

Each subject was positioned supine onto the MRI scanner bed and placed into a 3 tesla MRI scanner (Achieva, Philips Medical Systems), with the head immobilized in a 32-channel head coil to which padding was added to prevent head movement. A series of 180 gradient echo echo-planar image sets with blood oxygen level-dependent contrast were collected. Each image volume was positioned to cover the entire brain and extend caudally to include the caudal brainstem (37 axial slices; repetition time $=2000 \mathrm{~ms}$; echo time $=30 \mathrm{~ms}$; raw voxel size, $3.0 \times 3.0 \times 4.0 \mathrm{~mm}$ thick). In all 70 subjects, a high-resolution T1-weighted anatomical image of the entire brain was also collected (288 axial slices; repetition time $=5600 \mathrm{~ms}$; raw voxel size, $0.87 \times 0.87 \times 0.87 \mathrm{~mm}$ thick $)$.

\section{MRI and statistical analysis}

Image preprocessing

Using SPM12 (Friston et al., 1995) and custom software, fMRI images were realigned and movement parameters examined to ensure no subject displayed $>1 \mathrm{~mm}$ volume-to-volume movement in the $X, Y$, and $Z$ planes and 0.05 radians in the pitch, roll, and yaw directions. Physiological (i.e., cardiovascular and respiratory) noise was modeled and removed using the drifter toolbox (Särkkä et al., 2012). The fMRI images were detrended to remove global signal-intensity changes and then coregistered to the same subject's T1-weighted anatomical image. The T1-weighted image was then spatially normalized to the Montreal Neurological Institute (MNI) template. The normalization parameters were then applied to the fMRI images. This process resulted in the fMRI images being resliced into $2 \times 2 \times 2 \mathrm{~mm}$ voxels. To improve brainstem normalization accuracy, the brainstems of the fMRI image sets were isolated using the SUIT (A Spatially Unbiased Atlas Template of the 
Table 1. PTN subject characteristics

\begin{tabular}{|c|c|c|c|c|c|c|}
\hline Subject & $\begin{array}{l}\text { Age } \\
\text { (years) }\end{array}$ & Gender & Site & $\begin{array}{l}\text { Pain intensity } \\
\text { (visual analog scale) }\end{array}$ & $\begin{array}{l}\text { Pain duration } \\
\text { (months) }\end{array}$ & Medications \\
\hline 1 & 56 & Male & Right lower jaw & 2.4 & 72 & Amitriptyline \\
\hline 2 & 45 & Female & Bilateral (left jaw and temple) & 4.8 & 132 & Carbamazepine \\
\hline 3 & 45 & Female & Left upper jaw & 4.5 & 42 & None \\
\hline 4 & 42 & Female & Bilateral lower jaw & 4.0 & 42 & Paracetamol, ibuprofen \\
\hline 5 & 67 & Female & Left upper jaw and temple & 8.4 & 168 & None \\
\hline 6 & 43 & Male & Left upper jaw and temple & 5.8 & 192 & Desvenlafaxine \\
\hline 7 & 44 & Female & Bilateral upper and lower jaw & 1.2 & 18 & Ibuprofen, codeine phosphate, paracetamol \\
\hline 8 & 65 & Female & Left face and neck & 2.5 & 84 & Amitriptyline, paracetamol \\
\hline 9 & 50 & Female & Right lower jaw & 0.1 & 84 & None \\
\hline 10 & 67 & Female & Right upper jaw & 3.9 & 240 & $\begin{array}{l}\text { Thyroxine sodium, rosuvastatin, venlafaxine, } \\
\text { pregabalin, phenylethylamine, ergotamine }\end{array}$ \\
\hline 11 & 27 & Female & Left upper jaw & 6.9 & 29 & Ibuprofen, duloxetine \\
\hline 12 & 65 & Male & Bilateral: left lower and right upper jaw & 0.4 & 26 & Amitriptyline \\
\hline 13 & 38 & Female & Left lower jaw & 3.4 & 96 & Gabapentin, amitriptyline \\
\hline 14 & 45 & Female & Bilateral jaw & 2.8 & 17 & Ibuprofen \\
\hline 15 & 19 & Male & Right upper jaw & 5.0 & 88 & None \\
\hline 16 & 59 & Female & Left lower jaw & 4.4 & 115 & None \\
\hline 17 & 59 & Female & Left upper jaw & 4.4 & 120 & None \\
\hline 18 & 27 & Male & Left upper jaw and under eye & 3.6 & 8 & Palmitoylethanolamide, paracetemol, benzodiazepines \\
\hline 19 & 71 & Female & Left upper jaw & 5.1 & 9 & Meloxicam, paracetamol, gabapentin, nortriptyline \\
\hline 20 & 37 & Male & Left upper jaw and under eye & 2.0 & 84 & Oxycodone, gabapentin, paracetamol \\
\hline 21 & 35 & Female & Bilateral upper jaw & 6.1 & 27 & Flavonoids, paracetamol \\
\hline 22 & 27 & Male & Right upper and lower jaw & 0.5 & 41 & Palmitoylethanolamide, sertraline \\
\hline 23 & 21 & Female & Right upper jaw & 1.3 & 144 & Palmitoylethanolamide \\
\hline 24 & 35 & Male & Right upper jaw & 1.5 & 96 & Palmitoylethanolamide \\
\hline
\end{tabular}
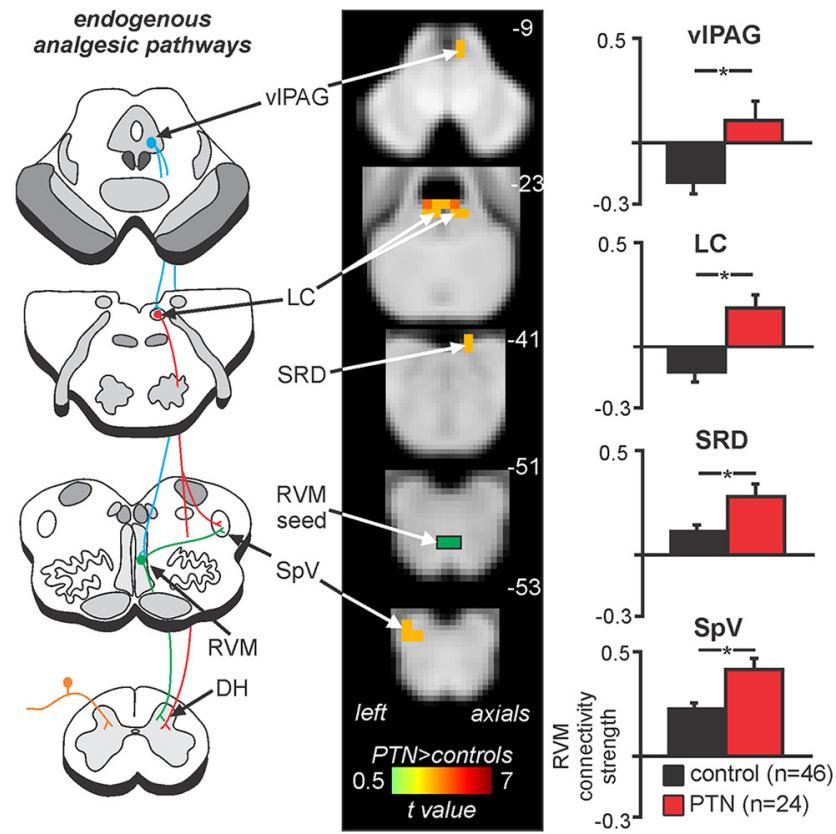

Figure 2. Regions within the brainstem endogenous analgesic pathways that show significantly greater RVM functional connectivity in subjects with PTN compared with pain-free controls. Left, Diagram of the endogenous analgesic pathways that modulate nociceptive transmission at the spinal dorsal horn (DH) and SpV. Yellow, Nociceptor afferent projection; blue, efferent projections from vIPAG; red, efferent projections from LC; green, efferent projections from RVM. Middle, Regions with enhanced RVM functional connectivity in subjects with PTN (hot color scale) overlaid onto template axial T1-weighted images. Slice locations in MNI space are indicated at the top right of each slice. The location of the RVM seed is indicated in green. Subjects with PTN show an increase in RVM connectivity with the right vIPAG, bilateral $\mathrm{LC}$, right SRD, and left SpV. Right, Bar graphs of mean ( \pm SEM) RVM connectivity strengths with significant clusters identified in the voxel-by-voxel analysis. ${ }^{*}$, Significant difference determined in the voxel-by-voxel analysis. Connectivity strength in arbitrary units.
Table 2. Location of clusters in MNI space, $t$ values, and sizes of clusters that had significantly different resting RVM and LC connectivity strengths in subjects with PTN compared to control subjects

\begin{tabular}{|c|c|c|c|c|c|}
\hline & \multicolumn{3}{|c|}{ MNI coordinates } & \multirow[b]{2}{*}{$t$ value } & \multirow[b]{2}{*}{ Cluster size } \\
\hline & $x$ & Y & Z & & \\
\hline \multicolumn{6}{|l|}{ RVM connectivity } \\
\hline \multicolumn{6}{|l|}{ PTN $>$ Control } \\
\hline $\begin{array}{l}\text { Right vIPAG } \\
\text { LC }\end{array}$ & $\mathrm{LC}$ & -32 & -9 & 2.72 & 9 \\
\hline Right & 4 & -38 & -23 & 3.92 & 24 \\
\hline Left & -2 & -36 & -21 & 3.67 & \\
\hline Right SRD & 6 & -44 & -41 & 2.81 & 2 \\
\hline Left SpV & -6 & -42 & -53 & 3.22 & 6 \\
\hline \multicolumn{6}{|c|}{ Right LC connectivity } \\
\hline \multicolumn{6}{|c|}{ PTN $>$ Control } \\
\hline Left SRD & 4 & -44 & -55 & 2.71 & 1 \\
\hline RVM & 0 & -36 & -47 & 3.11 & 6 \\
\hline \multicolumn{6}{|l|}{ Control > PTN } \\
\hline Right vIPAG & 6 & -28 & -5 & 3.10 & 28 \\
\hline
\end{tabular}

Cerebellum and Brainstem) toolbox in native space (Diedrichsen, 2006). Binary masks of the brainstem were created and these masks were then used to spatially normalize each image set to the SUIT brainstem template in MNI space. The resulting normalized fMRI images remained unsmoothed so as to maintain spatial accuracy of small brainstem structures.

Resting functional connectivity analysis

Voxel-by-voxel analysis. We performed brainstem-only functional connectivity analyses using the RVM region as the seed. Our previous investigations have shown that the nucleus raphe magnus within the RVM region displays anatomical changes in individuals with chronic pain (Wilcox et al., 2015b). The RVM seed comprised six contiguous voxels: two voxels each at three rostrocaudal levels from $z$ coordinate -53 to -49 in MNI space (Fig. 1). In each subject, signal intensity changes were extracted from this RVM seed and voxel-by-voxel analyses were per- 
formed to determine which brainstem areas displayed significant signal-intensity covariations with the RVM. The movement parameters derived from the realignment step were included as nuisance variables. The connectivity maps were smoothed using a $2 \mathrm{~mm}$ fullwidth half-maximum Gaussian filter and then placed into a second-level random-effects procedure to determine significant differences in RVM connectivity strength in arbitrary units, (A.U.), between PTN and control subjects. Following an initial uncorrected threshold of $p<$ 0.001, small-volume corrections were applied on the PAG, RVM, LC, SRD, and SpV using 40 $\mathrm{mm}^{3}$ hyper-rectangles centered at the location of each region based on the Duvernoy Brainstem Atlas (Naidich et al., 2009).

The resulting clusters of significant difference were used to extract connectivity strength values in each subject, and the mean $( \pm$ SEM) values were plotted to provide a measure of connectivity direction. In PTN subjects, we investigated the significance of relationships between RVM connectivity strengths and pain characteristics (i.e., pain intensity and duration) using regression analyses (Pearson's $r$, $p<0.05$ ).

The brainstem RVM connectivity analysis resulted in a significant cluster in the region of the LC. Since this region is known to be involved in nociceptive modulation (Taylor and Westlund, 2017), we also explored the resting connectivity of this brainstem region. The LC seed comprised six contiguous voxels: two voxels each at three rostrocaudal levels from $z$ coordinate -23 to -19 in MNI space (Fig. 1). We performed a brainstem resting connectivity analysis of the right LC in the same way as that described above for the RVM. Additionally, in PTN subjects, the significance of relationships between LC connectivity strengths and pain characteristics (i.e., pain intensity and duration) were determined for significant clusters using regression analyses (Pearson's $r, p<0.05$ ).

In addition to brainstem-specific analyses, resting connectivity strengths from the PAG and LC to higher brain regions were explored. Resting signal intensities from the right LC seed used for the brainstem analysis and from a right PAG seed (six voxels in size, derived from RVM connectivity analysis and spread across $z$ coordinates $-11,-9,-7$ ) were extracted and voxel-by-voxel analyses performed to determine connectivity strengths with higher brain regions. Movement parameters were included as nuisance variables and the resultant connectivity maps were smoothed using a $6 \mathrm{~mm}$ full-width half-maximum Gaussian filter. These connectivity maps were placed into a second-level random-effects procedure to determine significant differences in connectivity strengths between PTN and control subjects. Following an initial uncorrected threshold of $p<0.001$, we applied small-volume corrections (familywise error, $p<0.05$ ). The resulting clusters of significant difference were used to extract connectivity strength values in each subject and the mean $( \pm \mathrm{SEM})$ values plotted to provide a measure of connectivity direction. In PTN subjects, the significance of relationships between LC and PAG connectivity strengths and pain characteristics (i.e., pain intensity and duration) were determined for significant clusters using regression analyses (Pearson's $r, p<0.05$ ).

Subdivision analysis. To determine whether there are variations in rostrocaudal connectivity strengths within the vlPAG and SpV, we examined resting functional connectivity between these regions and both the RVM and LC. Rostrocaudal subdivisions of the vlPAG and SpV were created based on our voxel-by-voxel analyses and the Duvernoy Human Brainstem Atlas (Naidich et al., 2009). For the vlPAG, five four-voxel volumes of interest were created along the rostrocaudal axis on the left and right sides, extending from the caudal vlPAG at $z$ coordinate -13 , rostrally to $z$ coordinate -5 (Fig. 1). Similarly, for the $\mathrm{SpV}, 14$ four-voxel volumes of interest were created along the rostrocaudal axis on the left and right sides, extending from the caudal $\mathrm{SpV}$ at $z$ coordinate -61 , rostrally to $z$ coordinate -35 . RVM and LC connectivity strengths of these vlPAG and $\mathrm{SpV}$ volumes of interest were extracted and differences between controls and PTN subjects determined $(p<0.05$, two-sample $t$ test, Bonferroni corrected for multiple comparisons). Two PTN subjects were excluded from the SpV subdivision analyses due to their fMRI images not covering the entire caudal brainstem at $z$ level -61 .

\section{Results}

\section{Pain characteristics}

Individual PTN subject characteristics are shown in Table 1. All 24 PTN subjects were classified as having post-traumatic neuropathy. In 19 PTN patients, chronic pain was unilateral (7 right, 12 left), and the remaining five PTN subjects reported bilateral pain. In all PTN subjects, pain was located in the second and third trigeminal nerve divisions, with one patient also reporting pain in the first division. Their mean ( \pm SEM) pain intensity was $3.5 \pm 0.4$ of 10 and their mean duration of pain was $82.2 \pm 12.5$ months. Using the MPQ, PTN subjects most frequently described their pain as throbbing (58\%), shooting (54\%), and nagging (67\%).

\section{Brainstem resting functional connectivity}

$R V M$ voxel-by-voxel analysis

The brainstem voxel-by-voxel analysis revealed several key areas that display altered resting RVM functional connectivity in PTN subjects compared with controls (Fig. 2, Table 2). PTN subjects showed a significant increase in RVM connectivity strength in the region encompassing the right vlPAG (mean \pm SEM functional connectivity, a.u.: control, $-0.19 \pm 0.05$; PTN, $0.11 \pm 0.09$ ), the region of the LC bilaterally (control, $-0.12 \pm 0.04$; PTN, $0.18 \pm$ 0.07 ), the right dorsomedial medulla in the area of the SRD (con- 
trol, $0.11 \pm 0.03$; PTN, $0.29 \pm 0.06)$, and in the region encompassing the left SpV (control, $0.23 \pm 0.03$; PTN, $0.42 \pm 0.05$ ). In no brainstem region did controls show significantly greater RVM connectivity than PTN patients. In PTN subjects, there were no significant correlations between RVM connectivity strength in these brainstem regions and pain intensity (vlPAG: $r=0.21$; LC: $r=0.11$; SRD: $r=0.15$; SpV: $r=0.03$, all $p$ 's $>0.05)$ or pain duration (vlPAG: $r=0.18$; LC: $r=0.05$; SRD: $r=0.19$; SpV: $r=$ 0.38 , all $p$ 's $>0.05)$.

\section{$R V M$ to vlPAG and SpV subdivision analysis}

The subdivision analysis of RVM connectivity over the rostrocaudal extent of the bilateral vlPAG revealed that PTN subjects showed a trend toward higher RVM connectivity. However, this did not reach significance at the threshold we set (Fig. 3). In no vlPAG subdivision did controls show greater RVM connectivity than PTN subjects. In contrast, RVM-SpV subdivision analysis revealed that PTN subjects displayed greater connectivity strength with the left SpV (Fig. 3). This significantly greater RVM connectivity strength was restricted to the $\mathrm{SpV}$ within the region of the subnucleus interpolaris (SpVi; $z-53$ : control, $0.24 \pm 0.03$; PTN, $0.46 \pm 0.06, p<0.001)$ and subnucleus oralis (SpVo; $z-51$ : control, $0.27 \pm 0.03 ; \mathrm{PTN}, 0.46 \pm 0.06, p=0.002)$. There were no differences between groups in RVM-SpV connectivity strength on the right side and, again, in no $\mathrm{SpV}$ region did controls show greater connectivity than PTN subjects.

\section{LC voxel-by-voxel analysis}

The brainstem voxel-by-voxel analysis revealed two brainstem regions in which PTN subjects displayed greater LC connectivity compared with controls (Fig. 4, Table 2). Significantly greater LC connectivity in PTN subjects occurred within the left caudal SRD (control, $0.02 \pm 0.03$; PTN, $0.21 \pm 0.06$ ) and in the region of the RVM (control, $-0.06 \pm 0.05 ; \mathrm{PTN}, 0.21 \pm 0.06$ ). In contrast, PTN subjects showed significantly reduced LC connectivity strength with the right rostral vlPAG (control, $0.21 \pm 0.04$; PTN, $-0.03 \pm 0.05)$. In PTN subjects, there were no significant correlations between LC connectivity strength in these three brainstem regions and pain intensity (SRD: $r=0.26$; RVM: $r=0.06$; vlPAG: $r=0.33$; all $p$ 's $>0.05$ ) or pain duration (SRD: $r=0.11$; RVM: $r=0.12$; vlPAG: $r=0.21$; all $p$ 's $>0.05)$.

\section{LC to vlPAG and SpV subdivision analysis}

The subdivision analysis revealed that, in direct contrast to the RVM, LC connectivity strength with the right vlPAG was significantly reduced in PTN subjects (Fig. 5). PTN subjects showed significantly reduced LC connectivity strength in a discrete region of the rostral vlPAG $(z-5$ : control, $0.25 \pm 0.05$; PTN, $-0.04 \pm 0.07, p<0.001)$. There were no differences between groups in LC-vlPAG connectivity strength on the left side and in no vlPAG region did controls show greater LC connectivity than PTN subjects. In contrast, the LC-SpV subdivision analysis revealed that PTNs did not differ from controls in LC connectivity strength at any region of the $\mathrm{SpV}$.

\section{Whole-brain resting functional connectivity}

\section{$P A G$ voxel-by-voxel analysis}

The whole-brain voxel-by-voxel analysis revealed several key areas that display altered resting vlPAG functional connectivity in PTN subjects compared with controls (Fig. 6, Table 3). PTN subjects showed a significant increase in vlPAG connectivity strength in the region of the left nucleus accumbens (NAc; control, $-0.02 \pm 0.03$; PTN, $0.16 \pm 0.04)$, the bilateral posterior

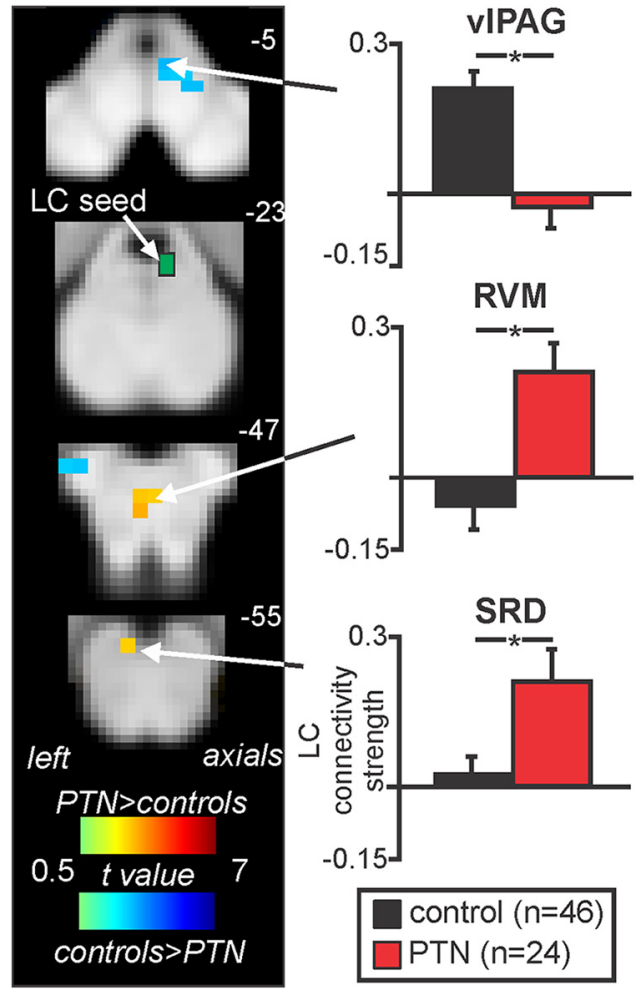

Figure 4. Regions within the brainstem pain-modulation system in which subjects with PTN show altered LC functional connectivity compared with pain-free controls. Areas with enhanced (hot color scale) and reduced (cool color scale) LC connectivity in PTN subjects are overlaid onto template axial T1-weighted images. Slice locations in MNI space are indicated at the top right of each slice, with the location of the LC seed indicated in green. Subjects with PTN showed an increase in LC connectivity with the left caudal SRD and the RVM, as well as a decrease in LC connectivity with the right vIPAG. Right, Bar graphs of mean ( \pm SEM) LC connectivity strengths with significant clusters identified in the voxel-by-voxel analysis. * , Significant difference determined in the voxel-by-voxel analysis. Connectivity strength in arbitrary units.

hippocampus (left: control, $-0.01 \pm 0.03$; PTN, $0.18 \pm 0.05$; right: control, $0.003 \pm 0.02 ; \mathrm{PTN}, 0.17 \pm 0.06)$, the bilateral thalamus in the region of the mediodorsal nucleus (left: control, $0.02 \pm 0.04 ; \mathrm{PTN}, 0.26 \pm 0.06$; right: control, $-0.03 \pm 0.03 ; \mathrm{PTN}$, $0.17 \pm 0.06$ ), and in the bilateral anterior hippocampus (left: control, $-0.01 \pm 0.03$; PTN, $0.24 \pm 0.06$; right: control, $-0.01 \pm$ 0.02 ; PTN, $0.18 \pm 0.04)$. In PTN subjects, there were no significant correlations between vlPAG connectivity strength in these brain regions and pain intensity (NAc, $r=0.09$; left posterior hippocampus, $r=0.32$; right posterior hippocampus, $r=0.027$; left thalamus, $r=0.04$; right thalamus, $r=0.27$; left anterior hippocampus, $r=0.15$; right anterior hippocampus, $r=0.08$; all $p$ 's $>0.05$ ) or pain duration (NAc, $r=0.31$; left posterior hippocampus, $r=0.21$; right posterior hippocampus, $r=0.20$; left thalamus, $r=0.14$; right thalamus, $r=0.09$; left anterior hippocampus, $r=0.17$; right anterior hippocampus, $r=0.06$; all p's >0.05).

LC voxel-by-voxel analysis

The whole-brain voxel-by-voxel analysis revealed several regions in which PTN subjects displayed greater LC connectivity compared with controls (Fig. 6, Table 3). Significantly greater LC connectivity in PTN subjects occurred within the left insula (control, $-0.001 \pm 0.02$; PTN, $0.11 \pm 0.04$ ), the left NAc (control, $0.003 \pm 0.03$; PTN, $0.16 \pm 0.04)$, the right anterior cingulate cortex (ACC; control, $0.02 \pm 0.01 ; \mathrm{PTN}, 0.08 \pm 0.02$ ), and within the bilateral frontal cortex (left: control, $0.02 \pm 0.02$; PTN, $0.15 \pm$ 
0.03; right: control, $0.002 \pm 0.02$; PN, $0.13 \pm 0.04)$. In PTN subjects, there was a significant positive correlation between LC-ACC connectivity strength and pain intensity $(r=0.42, p=0.047)$. That is, the greater the pain intensity, the greater the LC-ACC connectivity strength. No other significant correlations were observed.

\section{Discussion}

The results of this study demonstrate that PTN, a chronic neuropathic pain condition, is associated with ongoing functional alterations within the brainstem endogenous pain-modulation circuitry. Specifically, PTN patients showed increased functional connectivity between the RVM and the vlPAG, SRD, and LC, as well as with the brainstem region that receives orofacial nociceptor afferents, the SpV. Furthermore, PTN subjects showed increased functional connectivity strengths between these brainstem circuits and higher brain regions, such as the hippocampus, NAc, and ACC. These data show that chronic neuropathic pain is associated with ongoing functional changes in brainstem pain-modulation circuits that are likely involved in maintaining pain following nerve injury.

It is well known from experimental animal investigations that the vlPAG can evoke an opioid-mediated analgesia and that this effect is mediated by a projection to the spinal dorsal horn and SpV via the RVM (Basbaum and Fields, 1984; Fields and Heinricher, 1985; Bandler and Shipley, 1994). While it is well known that the RVM can inhibit incoming noxious information, it can also facilitate it and it has been suggested that opposing RVM inhibitory and facilitatory effects are finely balanced at rest in pain-free individuals (Fields and Heinricher, 1985; Heinricher et al., 2009; Ossipov et al., 2010). In contrast, in individuals with neuropathic pain, it has been proposed that this brainstem circuitry shifts to favor pronociceptive processes that contribute significantly to the maintenance of chronic pain (Pertovaara et al., 1996; Burgess et al., 2002; Vera-Portocarrero et al., 2006; Wang et al., 2013). Indeed, in rodents, deactivation of the PAG or RVM eliminates the hyperalgesia, allodynia, and spontaneous pain evoked by spinal nerve ligation (Pertovaara et al., 1996; Burgess et al., 2002; Wang et al., 2013). This functional shift appears to influence only the primary synapse ipsilateral to the side of nerve injury, since these analgesic effects are produced following interruption of the ipsilateral but not the contralateral RVMdorsal horn pathway (Wang et al., 2013). Furthermore, this pronociceptive shift in RVM function appears to be strictly related to the presence of pain behaviors and not to the nerve injury itself (De Felice et al., 2011).

The increased resting functional coupling between between the PAG and RVM and between the RVM and SpV reported in neuropathic pain subjects in this study is consistent with these preclinical findings and may reflect a state of continuous descending facilitation of nociceptive transmission at the $\mathrm{SpV}$ driven by the vlPAG via the RVM. Interestingly, in PTN subjects we found increased RVM connectivity specifically within the transition between the interpolaris and oralis regions of the left
SpV. This was expected given that the majority of our PTN subjects reported left-sided pain and the location of these functional coupling changes overlaps with our previous reports of structural changes at the SpVi and SpVo in PTN patients (Wilcox et al., 2015a).

Alongside the vlPAG and RVM, the LC can also directly and indirectly modulate nociceptive transmission at the dorsal horn and SpV (Sasa et al., 1979; Jones and Gebhart, 1986; Tsuruoka et al., 2003; Pertovaara, 2006; Kaushal et al., 2016). The LC has reciprocal connections with the vlPAG and the SpV (Sasa and Takaori, 1973; Jones and Moore, 1977; Cedarbaum and Aghajanian, 1978; Mantyh, 1983), and indeed we found a decrease in right LC connectivity strength with the rostral region of the ipsilateral vlPAG. However, we found no difference in LC connectivity strength with the SpV in the PTN subjects. This is surprising, since facilitation by the noradrenergic system contributes to mechanical hypersensitivity in animal models of neuropathic pain (Brightwell and Taylor, 2009; Kaushal et al., 2016). However, it has been proposed that the LC inhibitory effects are engaged through a direct connection to the dorsal horn and SpV (Sasa et al., 1974), whereas LC pronociceptive effects may be mediated via the SRD (Martins et al., 2013; Taylor and Westlund, 2017). Our results are consistent with this idea, as PTN subjects displayed enhanced LC-SRD connectivity strength and no change in LC-SpV connectivity strength. Alongside a decrease in LC-vlPAG connectivity, we found that PTN subjects display greater LC-RVM connectivity strength than controls, raising the prospect that the endogenous opioid and noradrenergic pain modulation systems interact differently in individuals with neuropathic pain. Although tract-tracing studies have shown that direct connections between the RVM and LC are modest (Clark and Proudfit, 1991), it has been demonstrated that the vlPAG-RVM and noradrenergic pain systems interact in experimental pain states to produce inhibitory effects at the dorsal horn (Aimone et al., 1987). 


\section{vIPAG connectivity}
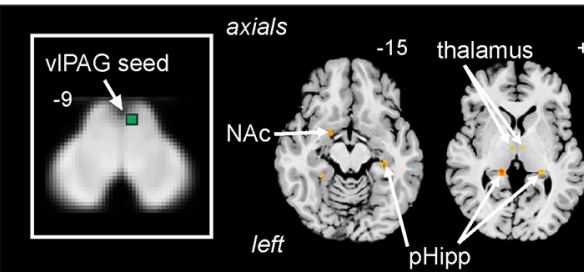

\section{coronal}
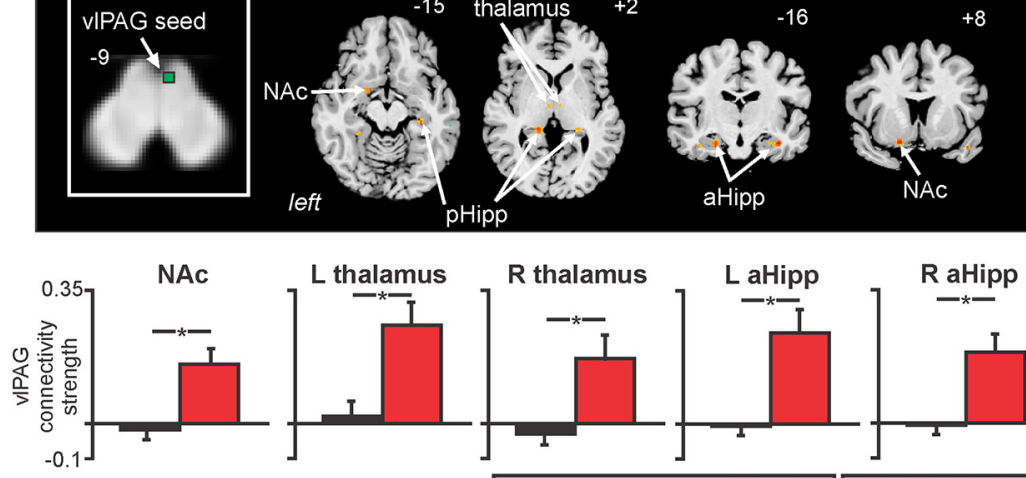

$\mathrm{R}$ thalamus

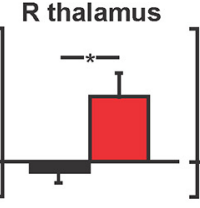

\section{L aHipp}

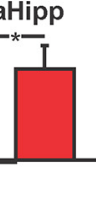

2 t value $5 \quad 2$ t value
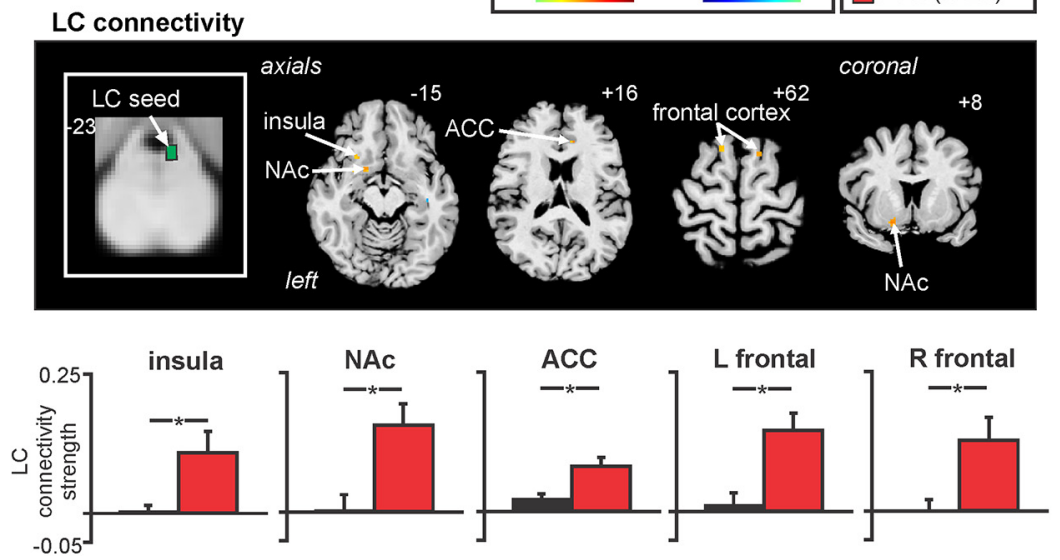

Figure 6. Rostral brain regions in which subjects with PTN show increased vIPAG and LC functional connectivity compared with healthy controls. Areas in which subjects with PTN display enhanced vIPAG or LC connectivity (hot color scale) are overlaid onto template axial and coronal T1-weighted images. Slice locations in MNI space are indicated at the top right of each slice, with the location of vIPAG and LC seeds indicated in green on the far left. Top, Subjects with PTN show enhanced vIPAG connectivity with the left NAc, bilateral posterior hippocampus (pHipp), bilateral anterior hippocampus (aHipp), and bilateral thalamus. Bottom, Subjects with PTN show increased LC connectivity with the left insula and NAC, right ACC, and bilateral frontal cortex. Below brain slices, plots of mean ( \pm SEM) vIPAG and LC connectivity strengths in significant clusters identified in the voxel-by-voxel analysis. * Significant difference determined in the voxel-by-voxel analyses. Connectivity strength in arbitrary units.

Table 3. Location of whole-brain clusters in MNI space, $t$ values, and sizes of clusters that had significantly different resting vIPAG and LC connectivity strengths in subjects with painful PTN compared to control subjects

\begin{tabular}{|c|c|c|c|c|c|}
\hline & \multicolumn{3}{|c|}{ MNI coordinates } & \multirow[b]{2}{*}{$t$ value } & \multirow[b]{2}{*}{ Cluster size } \\
\hline & $x$ & $y$ & $Z$ & & \\
\hline \multicolumn{6}{|l|}{ Right vIPAG connectivity } \\
\hline \multicolumn{6}{|l|}{ PTN $>$ Control } \\
\hline Left NAC & -18 & 2 & -16 & 3.44 & 25 \\
\hline Posterior hippocampus & -14 & -36 & 6 & 4.29 & 43 \\
\hline Left & 22 & -36 & 4 & 3.29 & 3 \\
\hline Right & 30 & -30 & -12 & 3.98 & 14 \\
\hline \multicolumn{6}{|l|}{ Thalamus } \\
\hline Left & -6 & -12 & 0 & 3.30 & 9 \\
\hline Right & 6 & -12 & 2 & 3.10 & 3 \\
\hline Anterior hippocampus & -24 & -14 & -24 & 4.17 & 67 \\
\hline Left & 36 & -16 & -22 & 4.17 & 50 \\
\hline Right & 26 & -12 & -20 & & \\
\hline \multicolumn{6}{|l|}{ Right LC connectivity } \\
\hline \multicolumn{6}{|l|}{ PTN $>$ Control } \\
\hline Left insula & -24 & 20 & -12 & 3.26 & 8 \\
\hline Left NAC & -18 & 8 & -16 & 3.47 & 17 \\
\hline Right ACC & 12 & 32 & 16 & 3.41 & 2 \\
\hline \multicolumn{6}{|l|}{ Frontal cortex } \\
\hline Left & -14 & 16 & 62 & 3.11 & 7 \\
\hline Right & 16 & 12 & 62 & 3.37 & 13 \\
\hline
\end{tabular}

We cannot infer directionality from functional connectivity analyses, and thus ascending signal transmission may also contribute to the alterations in fMRI signal coupling observed in PTN patients. Indeed, there are ascending projections from the SpV to the PAG, RVM, and LC (Craig, 1992; Bandler and Shipley, 1994; Sugiyo et al., 2005). While these ascending influences may be important, we suggest that the changes in brainstem connectivities that occur in PTN subjects most likely represent changes in descending modulation of the SpV since previous investigations suggest that neuropathic pain can be maintained solely by central mechanisms, even if initiated by a peripheral nerve injury (Burgess et al., 2002; Henderson et al., 2013; Wang et al., 2013).

While the brainstem contains the neural circuitry necessary for pain modulation, this system can be engaged by higher brain regions (Heinricher et al., 2009). Interestingly, we found increased resting connectivity strengths between the PAG, the mediodorsal thalamus, and the hippocampus in PTN subjects compared with controls. While it is possible that these regions represent top-down modulation of brainstem circuits, it has been suggested that the midline thalamic nuclei, such as the mediodorsal nuclei, receive ascending communication from the PAG, which is transferred to the ventral hippocampus to influence pain-related behaviors (Ballesteros et al., 2014). Furthermore, the hippocampus sends extensive projections to the NAc (Viard et al., 2011). The NAc is involved in mediating reward processing and goal-directed behavior, two processes that are disrupted in individuals with chronic pain (Navratilova and Porreca, 2014; Navratilova et al., 2016). Our PTN patients also displayed enhanced LC connectivity with the NAc and ACC, two regions involved in the rewarding effects of pain relief (Navratilova et al., 2016). Indeed, the only significant linear relationship occurred between LC-ACC connectivity strength and pain intensity in PTN subjects.

Several limitations are worth noting. First, given the small size of brainstem nuclei and the limited spatial resolution of current functional human brain-imaging techniques, it is difficult to assign significant clusters to specific brainstem nuclei. This does not, however, negate the validity of our results when placed into context. That is, our brainstem clusters overlap with regions that have been shown in preclinical studies to be critical for the expression of analgesia and our a priori hypothesis was that the function of these regions would be altered in PTN subjects. Furthermore, we did not smooth our images so that we could maintain spatial accuracy, though all these factors do not eliminate the possibility that closely neighboring nuclei are responsible for the reported differences. Second, the brainstem is susceptible to physiological noise and movement-related artifacts. To limit the potential influence of these factors, we applied a physiological noise correction and included movement param- 
eters as nuisance variables. A further potential limitation is the use of unflipped brain images for the PTN subjects. Most subjects reported unilateral pain, making it unlikely in most cases to observe connectivity changes bilaterally. Finally, a possible limitation is that when reporting between-group differences, we used a statistical threshold that was not corrected for multiple comparisons. Although this may introduce type-II errors, we limited this possibility by applying small-volume corrections. As detailed above, since numerous experimental animal investigations have reported that the RVM, PAG, LC, and SRD are critically involved in analgesic responses, we strongly suggest that small-volume corrections of these brainstem regions are entirely appropriate. Despite these limitations, we are confident that the altered brainstem connectivity presented here represents a functional difference associated with the presence of chronic neuropathic pain.

Overall, our findings show that chronic neuropathic pain in humans is associated with ongoing functional alterations in the brainstem endogenous pain-modulation system. These findings complement a wealth of experimental animal data suggesting that the functioning of brainstem modulation systems shifts to promote the maintenance of pain behaviors following nerve injury (Burgess et al., 2002; Ossipov et al., 2010; Wang et al., 2013). Furthermore, it is likely that the resting functioning of these brainstem circuits sets the ongoing gain of nociceptive processing, and may promote maladaptive responses to experimental noxious stimuli observed in various pain conditions (King et al., 2009; Yarnitsky, 2010; Granovsky, 2013). Our findings also suggest that higher brain regions, such as the hippocampus, NAc, and ACC, interact with the brainstem modulation circuitry differently in patients with neuropathic pain, possibly reflecting a top-down engagement of the circuitry alongside altered rewardcircuit functioning in chronic pain states.

\section{References}

Aimone LD, Jones SL, Gebhart GF (1987) Stimulation-produced descending inhibition from the periaqueductal gray and nucleus raphe magnus in the rat: mediation by spinal monoamines but not opioids. Pain 31:123136. CrossRef Medline

Alshelh Z, Di Pietro F, Youssef AM, Reeves JM, Macey PM, Vickers ER, Peck CC, Murray GM, Henderson LA (2016) Chronic neuropathic pain: it's about the rhythm. J Neurosci 36:1008-1018. CrossRef Medline

Ballesteros CI, de Oliveira Galvão B, Maisonette S, Landeira-Fernandez J (2014) Effect of dorsal and ventral hippocampal lesions on contextual fear conditioning and unconditioned defensive behavior induced by electrical stimulation of the dorsal periaqueductal gray. PLoS One 9:e83342. CrossRef Medline

Bandler R, Shipley MT (1994) Columnar organization in the midbrain periaqueductal gray: modules for emotional expression? Trends Neurosci 17: 379-389. CrossRef Medline

Bandler R, Keay KA, Floyd N, Price J (2000) Central circuits mediating patterned autonomic activity during active vs passive emotional coping. Brain Res Bull 53:95-104. CrossRef Medline

Basbaum AI, Fields HL (1984) Endogenous pain control systems: brainstem spinal pathways and endorphin circuitry. Annu Rev Neurosci 7:309-338. CrossRef Medline

Brightwell JJ, Taylor BK (2009) Noradrenergic neurons in the locus coeruleus contribute to neuropathic pain. Neuroscience 160:174-185. CrossRef Medline

Burgess SE, Gardell LR, Ossipov MH, Malan TP Jr, Vanderah TW, Lai J, Porreca F (2002) Time-dependent descending facilitation from the rostral ventromedial medulla maintains, but does not initiate, neuropathic pain. J Neurosci 22:5129-5136. Medline

Cedarbaum JM, Aghajanian GK (1978) Afferent projections to the rat locus coeruleus as determined by a retrograde tracing technique. J Comp Neurol 178:1-16. CrossRef Medline

Clark FM, Proudfit HK (1991) Projections of neurons in the ventromedial medulla to pontine catecholamine cell groups involved in the modulation of nociception. Brain Res 540:105-115. CrossRef Medline

Craig AD (1992) Spinal and trigeminal lamina I input to the locus coeruleus anterogradely labeled with Phaseolus vulgaris leucoagglutinin (PHA-L) in the cat and the monkey. Brain Res 584:325-328. CrossRef Medline

De Broucker T, Cesaro P, Willer JC, Le Bars D (1990) Diffuse noxious inhibitory controls in man. Involvement of the spinoreticular tract. Brain 113:1223-1234. CrossRef Medline

De Felice M, Sanoja R, Wang R, Vera-Portocarrero L, Oyarzo J, King T, Ossipov MH, Vanderah TW, Lai J, Dussor GO, Fields HL, Price TJ, Porreca $F$ (2011) Engagement of descending inhibition from the rostral ventromedial medulla protects against chronic neuropathic pain. Pain 152:2701-2709. CrossRef Medline

Diedrichsen J (2006) A spatially unbiased atlas template of the human cerebellum. Neuroimage 33:127-138. CrossRef Medline

Fields HL, Heinricher MM (1985) Anatomy and physiology of a nociceptive modulatory system. Philos Trans R Soc Lond B Biol Sci 308:361-374. CrossRef Medline

Floyd NS, Price JL, Ferry AT, Keay KA, Bandler R (2000) Orbitomedial prefrontal cortical projections to distinct longitudinal columns of the periaqueductal gray in the rat. J Comp Neurol 422:556-578. CrossRef Medline

Friston KJ, Holmes AP, Worsley KP, Proline JB, Frith CD, Frackowiak RSJ (1995) Statistical parametric maps in functional imaging: a general imaging approach. J Hum Brain Mapp 2:189-210. CrossRef

Garrett PH, Sarlani E, Grace EG, Greenspan JD (2013) Chronic temporomandibular disorders are not necessarily associated with a compromised endogenous analgesic system. J Orofac Pain 27:142-150. CrossRef Medline

Granovsky Y (2013) Conditioned pain modulation: a predictor for development and treatment of neuropathic pain. Curr Pain Headache Rep 17: 361. CrossRef Medline

Gustin SM, Peck CC, Wilcox SL, Nash PG, Murray GM, Henderson LA (2011) Different pain, different brain: thalamic anatomy in neuropathic and non-neuropathic chronic pain syndromes. J Neurosci 31:5956-5964. CrossRef Medline

Heinricher MM, Tavares I, Leith JL, Lumb BM (2009) Descending control of nociception: specificity, recruitment and plasticity. Brain Res Rev 60: 214-225. CrossRef Medline

Henderson LA, Peck CC, Petersen ET, Rae CD, Youssef AM, Reeves JM, Wilcox SL, Akhter R, Murray GM, Gustin SM (2013) Chronic pain: lost inhibition? J Neurosci 33:7574-7582. CrossRef Medline

Jones BE, Moore RY (1977) Ascending projections of the locus coeruleus in the rat. II. Autoradiographic study. Brain Res 127:23-53. CrossRef Medline

Jones SL, Gebhart GF (1986) Quantitative characterization of ceruleospinal inhibition of nociceptive transmission in the rat. J Neurophysiol 56:13971410. Medline

Kaushal R, Taylor BK, Jamal AB, Zhang L, Ma F, Donahue R, Westlund KN (2016) GABA-A receptor activity in the noradrenergic locus coeruleus drives trigeminal neuropathic pain in the rat; contribution of NA $\alpha 1$ receptors in the medial prefrontal cortex. Neuroscience 334:148-159. CrossRef Medline

King CD, Wong F, Currie T, Mauderli AP, Fillingim RB, Riley JL 3rd (2009) Deficiency in endogenous modulation of prolonged heat pain in patients with irritable bowel syndrome and temporomandibular disorder. Pain 143:172-178. CrossRef Medline

Le Bars D, Dickenson AH, Besson JM (1979) Diffuse noxious inhibitory controls (DNIC). I. Effects on dorsal horn convergent neurones in the rat. Pain 6:283-304. CrossRef Medline

Mantyh PW (1983) Connections of midbrain periaqueductal gray in the monkey. II. Descending efferent projections. J Neurophysiol 49:582-594. Medline

Martins I, de Vries MG, Teixeira-Pinto A, Fadel J, Wilson SP, Westerink BH, Tavares I (2013) Noradrenaline increases pain facilitation from the brain during inflammatory pain. Neuropharmacology 71:299-307. CrossRef Medline

Mason P (2012) Medullary circuits for nociceptive modulation. Curr Opin Neurobiol 22:640-645. CrossRef Medline

Naidich TP, Duvernoy H. M., Delman B. N., Sorensen A. G., Kollias SS, M. HE (2009) Duvernoy's atlas of the human brain stem and cerebellum. Vienna: Springer/Wien.

Nasri-Heir C, Khan J, Benoliel R, Feng C, Yarnitsky D, Kuo F, Hirschberg C, Hartwell G, Huang CY, Heir G, Korczeniewska O, Diehl SR, Eliav E 
(2015) Altered pain modulation in patients with persistent postendodontic pain. Pain 156:2032-2041. CrossRef Medline

Navratilova E, Porreca F (2014) Reward and motivation in pain and pain relief. Nat Neurosci 17:1304-1312. CrossRef Medline

Navratilova E, Morimura K, Xie JY, Atcherley CW, Ossipov MH, Porreca F (2016) Positive emotions and brain reward circuits in chronic pain. J Comp Neurol 524:1646-1652. CrossRef Medline

Nurmikko TJ, Eldridge PR (2001) Trigeminal neuralgia-pathophysiology, diagnosis and current treatment. Br J Anaesth 87:117-132. CrossRef Medline

Ossipov MH, Dussor GO, Porreca F (2010) Central modulation of pain. J Clin Invest 120:3779-3787. CrossRef Medline

Pertovaara A (2006) Noradrenergic pain modulation. Prog Neurobiol 80: 53-83. CrossRef Medline

Pertovaara A (2013) The noradrenergic pain regulation system: a potential target for pain therapy. Eur J Pharmacol 716:2-7. CrossRef Medline

Pertovaara A, Wei H, Hämäläinen MM (1996) Lidocaine in the rostroventromedial medulla and the periaqueductal gray attenuates allodynia in neuropathic rats. Neurosci Lett 218:127-130. CrossRef Medline

Särkkä S, Solin A, Nummenmaa A, Vehtari A, Auranen T, Vanni S, Lin FH (2012) Dynamic retrospective filtering of physiological noise in BOLD fMRI: DRIFTER. Neuroimage 60:1517-1527. CrossRef Medline

Sasa M, Takaori S (1973) Influence of the locus coeruleus on transmission in the spinal trigeminal nucleus neurons. Brain Res 55:203-208. CrossRef Medline

Sasa M, Munekiyo K, Ikeda H, Takaori S (1974) Noradrenaline-mediated inhibition by locus coeruleus of spinal trigeminal neurons. Brain Res 80:443-460. CrossRef Medline

Sasa M, Fujimoto S, Igarashi S, Munekiyo K, Takaori S (1979) Microiontophoretic studies on noradrenergic inhibition from locus coeruleus of spinal trigeminal nucleus neurons. J Pharmacol Exp Ther 210:311-315. Medline

Sugiyo S, Takemura M, Dubner R, Ren K (2005) Trigeminal transition zone/rostral ventromedial medulla connections and facilitation of orofacial hyperalgesia after masseter inflammation in rats. J Comp Neurol 493:510-523. CrossRef Medline

Taylor BK, Westlund KN (2017) The noradrenergic locus coeruleus as a chronic pain generator. J Neurosci Res 95:1336-1346. CrossRef Medline

Tsuruoka M, Matsutani K, Inoue T (2003) Coeruleospinal inhibition of nociceptive processing in the dorsal horn during unilateral hindpaw inflammation in the rat. Pain 104:353-361. CrossRef Medline

Vera-Portocarrero LP, Xie JY, Yie JX, Kowal J, Ossipov MH, King T, Porreca F (2006) Descending facilitation from the rostral ventromedial medulla maintains visceral pain in rats with experimental pancreatitis. Gastroenterology 130:2155-2164. CrossRef Medline

Viard A, Doeller CF, Hartley T, Bird CM, Burgess N (2011) Anterior hippocampus and goal-directed spatial decision making. J Neurosci 31:46134621. CrossRef Medline

Wang R, King T, De Felice M, Guo W, Ossipov MH, Porreca F (2013) Descending facilitation maintains long-term spontaneous neuropathic pain. J Pain 14:845-853. CrossRef Medline

Wilcox SL, Gustin SM, Macey PM, Peck CC, Murray GM, Henderson LA (2015a) Anatomical changes at the level of the primary synapse in neuropathic pain: evidence from the spinal trigeminal nucleus. J Neurosci 35:2508-2515. CrossRef Medline

Wilcox SL, Gustin SM, Macey PM, Peck CC, Murray GM, Henderson LA (2015b) Anatomical changes within the medullary dorsal horn in chronic temporomandibular disorder pain. Neuroimage 117:258-266. CrossRef Medline

Yarnitsky D (2010) Conditioned pain modulation (the diffuse noxious inhibitory control-like effect): its relevance for acute and chronic pain states. Curr Opin Anaesthesiol 23:611-615. CrossRef Medline

Youssef AM, Macefield VG, Henderson LA (2016) Pain inhibits pain; human brainstem mechanisms. Neuroimage 124:54-62. CrossRef Medline 